\title{
Bistability, negative strain rate sensitivity and visualization of dislocation configurations
}

\section{G. Ananthakrishna*}

Materials Research Centre, Indian Institute of Science, Bangalore-560012, India

E-mail: garani@mrc.iisc.ernet.in

\begin{abstract}
Describing spatio-temporal features of the Portevin-Le Chatelier effect is known to be a difficult task as they emerge due to collective behavior of dislocations. In this article, we describe a dynamical approach to understand three aspects of the phenomenon, namely, the bistable nature of stress envelope, the negative strain rate sensitivity and visualization of dislocation configurations in different regimes of strain rate. We shall use some special dynamical techniques such as the reductive perturbative technique and slow manifold approach to understand these features. Using a reductive perturbative technique, we derive Stuart-Landau equation for the complex order parameter representing the amplitude and the phase of the limit cycle solution in the neighbourhood of the Hopf bifurcation. The slow manifold technique is used to map different parts of the slow manifold with the different branches of the negative SRS. More importantly, the method allows us to visualize the dislocation configurations in the regions of the bands.
\end{abstract}

International Conference on Statistical Mechanics of Plasticity and Related Instabilities August 29-September 2, 2005

Indian Institute of Science, Bangalore, India

${ }^{*}$ Speaker. 


\section{INTRODUCTION}

Describing spatio-temporal features of the Portevin-Le Chatelier (PLC) effect is a particularly diffi cult task for a variety of reasons. First, plastic deformation is a highly dissipative irreversible nonequilibrium process. Second, these spatio-temporal structures emerge from the collective behavior of dislocations in real materials, describing pattern formation in such realistic conditions is far more diffi cult than those nonequilibrium condensed matter situations which are relatively simple where experimental results correspond to ideal conditions. (Of course, one is making a continuous effort to obtain ideal experimental conditions.) Third, while describing properties of individual dislocations and their interactions have been known for a long time, there is no accepted framework to describe the collective properties of dislocations. Some of these diffi culties has been exposed in the efforts that have been pursued in the last three decades. One direction that the author has introduced and practiced for over two decades is to adopt nonlinear dynamical approach to understand the collective behavior of dislocations. In this article, we will focus on a few aspects of the PLC effect such as bistability, dynamical interpretation of negative strain rate sensitivity of the flow stress and visualization of dislocation confi gurations using slow manifold approach.

The Portevin-Le Chatelier (PLC) effect [2] refers to a kind of spatio-temporal instability observed when metallic alloys are deformed under constant strain rate confi tions. The temporal instability manifests itself in the form of serrations on the stress-strain curves [1,3]. Each stress drop is generally associated with the nucleation and often the propagation of a band of localized plastic deformation. Three types of bands have been identifi ed. With increasing strain rate or decreasing the temperature, one first fi nds the type $\mathrm{C}$ band, identifi ed with randomly nucleated static bands with nearly regular large characteristic stress drops. Then the type B 'hopping' bands are seen with serrations that are more irregular with amplitudes that are smaller than that for the type $\mathrm{C}$. The bands formed are still localized and static in nature, but forming ahead of the previous band in a spatially correlated way giving the visual impression of a hopping propagation. Finally, one observes the continuously propagating type A bands associated with small stress drops. These different types of PLC bands are believed to represent distinct correlated states of dislocations in the bands.

Conventional explanation of the PLC effect is based on the concept of dynamic strain aging (DSA) fi rst introduced by Cottrell [4] and later extended by others [1, 5, 7, 6]. In the Cotrell's picture, DSA refers to the interaction of mobile dislocations with the diffusing solute atoms. At low strain rates (or high temperatures) the average velocity of dislocations is low and there is suffi cient time for the solute atoms to diffuse to the dislocations and pin them (called as aging). Thus, longer the dislocations are arrested, larger will be the stress required to unpin them. When these dislocations are unpinned, they move abruptly till they are arrested again. At high strain rates (or low temperatures), the time available for solute atoms to diffuse to the dislocations decreases and hence the stress required to unpin them decreases. Thus, in a range of strain rates and temperatures where these two time scales are of the same order of magnitude, the PLC instability manifests. The competition between the slow rate of pinning and sudden unpinning of the dislocations, at the macroscopic level translates into a negative strain rate sensitivity (SRS) of the flow stress as a function of strain rate which is the basic instability mechanism used in most phenomenological models $[1,3]$. It may be pointed out that slow-fast dynamics and the negative flow rate characteristic is 
common to many stick-slip systems such as frictional sliding [8], fault dynamics [9] and peeling of an adhesive tape [10].

Among pattern forming systems the PLC effect is much harder to describe as it involves both fast and slow collective modes of dislocations. This requires specifi c techniques of nonlinear dynamics. Further, these time scales themselves evolve as a function of strain rate and temperature which in turn leads to different types of serrations. At low strain rates, the existence of both fast time scale corresponding to stress drops and the slow time scale corresponding to the loading time scale are clearly discernable. This also means the bistable nature of stress reflected in the upper and the lower envelope of the stress values. However, at high strain rate, as internal (plastic) relaxation is not complete, a clear demarcation of time scales becomes diffi cult. This along with the corresponding length scales (band widths), which also evolve, points to an extremely complex underlying dynamics.

The inherent nonlinearity and the presence of multiple time scales demands the use of tools and concepts of nonlinear dynamics for a proper understanding of this phenomenon. The fi rst dynamical approach was undertaken in early 80 s by Ananthakrishna and coworkers [11], which by its very nature affords a natural basis for the description of time dependent aspects of the PLC effect. Further, it also allows for explicit inclusion and interplay of different time scales inherent in the dynamics of dislocations [11]. Despite the simplicity of the model, many generic features of the PLC effect such as the existence of a window of strain rates and temperatures within which it occurs, etc., were correctly reproduced. More importantly, the negative SRS was shown to emerge naturally in the model, as a result of nonlinear interaction of the participating defects [11, 12].

Due to the dynamical nature of the model, one prediction that is unique to this model is the existence of the chaotic stress drops in a certain range of temperatures and strain rates [13]. This triggered a series of experiments to verify this prediction. The method followed was to analyze the stress-time series $[14,15]$ using dynamical methods [16, 17]. Apart from confi rming the chaotic nature of stress drops in a window of strain rates, these attempts have shown that a wealth of dynamical information can be extracted from the stress-time series obtained during the PLC effect $[14,15]$. Indeed, the number of degrees of freedom estimated from the experimental time series turn out to be same as in the model offering justifi cation for ignoring spatial degrees of freedom in the early investigations. Subsequent efforts to extend this analysis to the time series obtained over a range of strain rates showed an intriguing crossover from a chaotic state at low and medium strain rates to a power law state at high strain rates $[18,19]$. As the crossover is observed in both single and polycrystals, it appears to be insensitive to the microstructure. However, at a fundamental level, chaotic state is dynamically distinct from the power law state. The former involves a small number of degrees of freedom characterized by the self-similarity of the attractor and sensitivity to initial conditions [17] while latter is an infi nite dimensional state reminiscent of self-organized criticality (SOC) [20,21]. Due to this basic difference in the nature of the dynamics, most systems exhibit either of these states. More importantly, these studies also demonstrate that the nature of the dynamics in a given strain rate regime is correlated with the nature of band type. The chaotic state has been identifi ed with the type B bands and the scaling regime at high strain rate with the propagating type A bands [19]. The similarity between the transition from the static type B to the propagative type A bands with the Anderson's transition in condensed matter physics has been pointed out. Indeed, recently the spatio-temporal features of the PLC effect have attracted attention 
from physicists also [22]. Thus, it appears that the PLC effect is a storehouse of many paradigms in condensed matter physics.

The extension of the model to include spatial degrees of freedom has demonstrated that the above mentioned crossover detected in the analysis of experimental time series can be explained fully [23]. Further, the model exhibits the three types of bands as well. More importantly, a specifi c methodology used in understanding the crossover, namely the slow manifold approach helps to visualize dislocation confi gurations in the different band regimes of strain rates.

In this paper, we shall review three different aspects of the PLC effect within the scope of the Ananthakrishna's model (AK model for short). First the derivation of Stuart - Landau equation for the amplitude of serrations in the neighborhood of Hopf bifurcation to demonstrate the bistable nature of the stress. The second, we provide a dynamical interpretation of the negative strain rate sensitivity of the flow stress based on the structure of the slow manifold. Finally, using the same approach we show that visualization of dislocation confi gurations in the type $\mathrm{C}, \mathrm{B}$ and $\mathrm{A}$ band regimes is possible.

Section II, briefly introduces the dynamical model and its extension to include spatial degrees of freedom. Section III contains the numerical procedure used. In Section IV, we introduce the background material used for the study. Section V introduces the reductive perturbative approach and applies it to the model to obtain Ginzberg-Landau type of equation for the amplitude of the serrations. Section VI deals with a dynamical interpretation of the negative strain rate sensitivity (SRS) of the flow stress. Section V contains the slow manifold method of visual realization of dislocation confi gurations. We conclude the paper with a few general comments.

\section{THE ANANTHAKRSHANA's MODEL}

In the dynamical model due to Ananthakrishna and coworkers [11], the well separated time scales mentioned in the DSA are mimicked by three types of dislocations, namely, the fast mobile, immobile and the 'decorated' Cottrell type dislocations. The basic idea of the model is that all the qualitative features of the PLC effect emerge from the nonlinear interaction of these few dislocation populations, assumed to represent the collective degrees of freedom of the system. As the model has been studied in detail [24, 25, 26], following the notation in Ref. [12], we shall briefly outline the model in the scaled variables. The model consists of densities of mobile, immobile, and Cottrell's type dislocations denoted by $\rho_{m}(x, t), \rho_{i m}(x, t)$ and $\rho_{c}(x, t)$ respectively, in the scaled form. The evolution equations are:

$$
\begin{aligned}
\frac{\partial \rho_{m}}{\partial t} & =-b_{0} \rho_{m}^{2}-\rho_{m} \rho_{i m}+\rho_{i m}-a \rho_{m}+\phi_{e f f}^{m} \rho_{m}+\frac{D}{\rho_{i m}} \frac{\partial^{2}\left(\phi_{e f f}^{m}(x) \rho_{m}\right)}{\partial x^{2}} \\
\frac{\partial \rho_{i m}}{\partial t} & =b_{0}\left(b_{0} \rho_{m}^{2}-\rho_{m} \rho_{i m}-\rho_{i m}+a \rho_{c}\right) \\
\frac{\partial \rho_{c}}{\partial t} & =c\left(\rho_{m}-\rho_{c}\right)
\end{aligned}
$$

The model includes the following dislocation mechanisms: immobilization of two mobile dislocations due to the formation of locks $\left(b_{0} \rho_{m}^{2}\right)$, the annihilation of a mobile dislocation with an immobile one $\left(\rho_{m} \rho_{i m}\right)$, the remobilization of the immobile dislocation due to stress or thermal activation 
$\left(\rho_{\text {im }}\right)$. It also includes the immobilization of mobile dislocations due to solute atoms $\left(a \rho_{m}\right)$. Once a mobile dislocation starts acquiring solute atoms we regard it as the Cottrell's type dislocation $\rho_{c}$. As they progressively acquire more solute atoms, they eventually stop, then they are considered as immobile dislocations $\rho_{i m}$. Alternately, the aggregation of solute atoms can be regarded as the defi nition of $\rho_{c}$, ie., $\rho_{c}=\int_{-\infty}^{t} d t^{\prime} \rho_{m}\left(t^{\prime}\right) K\left(t-t^{\prime}\right)$, where $K(t)$ is an appropriate kernel. For the sake of simplicity, this kernel is modeled through a single time scale, $K(t)=e^{-c t}$. The convoluted nature of the integral physically implies that the mobile dislocations to which solute atoms aggregate earlier will be aged more than those which acquire solute atoms later (see ref. [12]). The fi fth term in Eqn.(1) represents the rate of multiplication of dislocations due to cross-slip. This depends on the velocity of the mobile dislocations taken to be $V_{m}(\phi)=\phi_{e f f}^{m}$, where $\phi_{e f f}=\left(\phi-h \rho_{i m}^{1 / 2}\right)$ is the scaled effective stress, $\phi$ the scaled stress, $m$ the velocity exponent and $h$ a work hardening parameter.

The nature of the spatial coupling in the PLC effect has been a matter of much debate [1]. Several mechanisms have been suggested as a source of spatial coupling [1]. Within the scope of our model, cross-slip is a natural source of spatial coupling, as dislocations generated due to cross slip at a point spread over to the neighboring elements. It is this that gives rise to the last term in Eq. 2.1 [23]. Note the factor $\rho_{\text {im }}^{-1}$ which models the fact that cross-slip spreads only into regions of minimum back stress. Finally, $a, b_{0}$ and $c$ are the scaled rate constants referring, respectively, to the concentration of solute atoms slowing down the mobile dislocations, the thermal and athermal reactivation of immobile dislocations, and the rate at which the solute atoms are gathering around the mobile dislocations. These equations are coupled to the machine equation

$$
\frac{d \phi(t)}{d t}=d\left[\dot{\varepsilon}-\frac{1}{l} \int_{0}^{l} \rho_{m}(x, t) \phi_{e f f}^{m}(x, t) d x\right]
$$

where $\dot{\varepsilon}$ is the scaled applied strain rate, $d$ the scaled effective modulus of the machine and the sample, and $l$ the dimensionless length of the sample. (We reserve $\dot{\varepsilon}_{a}$ for the unscaled strain rate.) We also note here that there is a feed back mechanism between the machine equation Eq. 2.4 and Eq.2.1.

\section{NUMERICAL SOLUTION OF THE MODEL}

We fi rst note that the spatial dependence of $\rho_{i m}$ and $\rho_{c}$ arises only through that of $\rho_{m}$. We solve the above set of equations by discretizing the specimen length into $N$ equal parts. Then, $\rho_{m}(j, t)$, $\rho_{\text {im }}(j, t), \rho_{c}(j, t), j=1, \ldots, N$, and $\phi(t)$ are solved. The widely differing time scales $[12,27,28]$ calls for appropriate care in the numerical solutions. The initial values of the dislocation densities are so chosen that they mimic the values in real samples. As for the boundary conditions, we note that the sample is strained at the grips. This means that there is a high density of immobile dislocations at the ends of the sample. We simulate this by employing two orders of magnitude higher values for $\rho_{i m}(j, t)$ at the end points $j=1$, and $N$ than the rest of the sample. Further, as bands cannot propagate into the grips, we use $\rho_{m}(j, t)=\rho_{c}(j, t)=0$ at $j=1$ and $N$.

As in the original model without spatial degrees of freedom, the PLC state is reached through a Hopf bifurcation and is terminated by a reverse Hopf bifurcation (with the other parameters kept in the instability domain). The number of complex conjugate roots are $2 \mathrm{~N}$, the negative ones 
are $\mathrm{N}$ and one zero exponent. The boundary of $\dot{\varepsilon}$ is approximately in the range 10 to 1000 for $a=0.8, b_{0}=0.0005, c=0.08, d=0.00006, m=3.0, h=0$ with $D=0.5$, beyond which a uniform steady state exits.

\section{Subspaces of slow and fast time scales}

As stated earlier, a proper description of the PLC effect requires a description of both the slow and fast time scales which in turn requires special techniques in nonlinear dynamics. These two time scales are transparent in the model equations where Eq. (2.1) represents a fast dynamics compared to the rest ( both Eq. (2.2) and (2.4) are slow while (2.3) falls in between). Such a system can be studied by eliminating the fast variable thereby allowing a reduction in the dimensionality of the system [29]. To illustrate this consider

$$
\begin{aligned}
\mu \dot{x} & =f(x, y, \mu), \\
\dot{y} & =g(x, y, \mu),
\end{aligned}
$$

where $\mu$ is small parameter and $x \in \mathbb{R}^{p}$ and $y \in \mathbb{R}^{q}$. The main feature of such systems is that $x$ evolves much faster than $y$ unless $f(x, y, \mu)$ is small. In the vicinity of the slow manifold defi ned by $f(x, y, \mu)=0$, the dynamics is characterized by the evolution of the slow variable $y$. Thus, there is a reduction in the dimensionality of the system. On the other hand, if one is interested in the fast sub-system, using a scaled time $\tau=t / \mu$, we get the corresponding fast variable $x$ defi ned by Eq. 4.1 where the slow variables $y$ act as parameters (obtained from Eq. 4). This subspace is clearly the complimentary subspace of the slow manifold. We shall use these the subspace spanned by the slow modes for the visualization of dislocation confi gurations and the complimentary subspace of fast variable to obtain the band velocity at high strain rates respectively [23].

\section{REDUCTIVE PERTURBATIVE APPROACH}

As mentioned in the introduction, in the regime of low strain rates (type C), two distinct levels of stress are discernable that correspond to the top and bottom of serrations. We fi rst attempt to understand the origin of this bistability in this model as no input of negative SRS feature has been incorporated. We shall shown that this arises due to Hopf bifurcation of the system of equations. For the sake of simplicity, we consider the creep case where the scaled stress is held constant and ignore the spatial inhomogeneous deformation.

We begin with a brief outline the reductive perturbative approach to problems of formation of new states of order in far-from-equilibrium situations. Transitions occurring in these systems are quite analogous to equilibrium phase transitions. The general idea is to construct a "potentiallike function" for the "order-parameter"-like variable in the neighborhood of the critical value of the drive parameter. This would permit the use of the methods developed in equilibrium phase transition for further analysis. Below the point of Hopf bifurcation of the system where the fi xed point is still stable, a pair of complex conjugate eigenvalues with real negative parts and another real negative eigenvalue exist for the linearized system of equations around the steady state. As we approach the critical bifurcation value from below, the real part of the pair of complex conjugate 
eigenvalues approaches zero from the negative side, and hence the corresponding eigendirections have a slow time scale. As we increase the drive parameter further, the real parts of the complex conjugate eigen values become positive and this region of the drive parameter is unstable. Thus, while the two eigenvectors corresponding to the pair of complex conjugate eigenvalues are slow modes, the eigenvector corresponding to the real negative eigenvalue is a fast (and decaying) mode. For this reason, the slow modes determine the formation of new states of order. The reductive perturbative method is a method where the slow enslaving dynamics is extracted in a systematic way $[30,31,32,33,34,35,36]$. The method involves fi rst fi nding the critical eigenvectors corresponding to the bifurcation point and expressing the general solution as a linear combination of these vectors. The effect of the nonlinearity is handled progressively using the multiple-scale method. The equation governing the complex order parameter takes the form of the Stuart-Landau equation, and corresponds to the time-dependent Ginzburg-Landau equation for a homogeneous medium. On the other hand, the asymptotic solution, which is a limit cycle, collapses to the subspace spanned by the slow modes with no trace of the fast mode. There are number of equivalent methods including reduction to the center manifold $[35,37,38]$.

We first scale out the stress variable by defining $\tau=\phi^{n} t$ and redefine $y=\rho_{m} / \phi^{m}, x=\rho_{m}$ $z=\rho_{c}$, and all the parameters $a, b_{0}$ and $c$ in the following are scaled by $\phi^{m}$. The equations for the mobile, immobile and Cottrel type dislocations are

$$
\begin{aligned}
& \dot{x}=(1-a) x-b_{0} x^{2}-x y+y, \\
& \dot{y}=b_{0}\left(b_{0} x^{2}-x y+a z-y\right), \\
& \dot{z}=c(x-z),
\end{aligned}
$$

In terms of new variables which are deviations from the fi xed point $\left(x_{a}, y_{a}, z_{a}\right)$, namely $X=x-$ $x_{a}, Y=y-y_{a}$, and $Z=z-z_{a}$, Eqs (5.1-5.3) take the form

$$
\begin{aligned}
& \dot{X}=-\left(\alpha X+\chi Y+b_{0} X^{2}+X Y\right), \\
& \dot{Y}=-b_{0}\left(\Gamma X+\delta Y-a Z-b_{0} X^{2}+X Y\right), \\
& \dot{Z}=c(X-Z),
\end{aligned}
$$

where

$$
\begin{array}{r}
\alpha=a+2 b_{0} x_{a}+y_{a}-1, \quad \chi=x_{a}-1, \\
\Gamma=y_{a}-2 b_{0} x_{a}, \quad \delta=x_{a}+1 .
\end{array}
$$

Equations (5.4)-(5.6) will be solved using reductive perturbative method. Writing these equations as a matrix equation where the nonlinear part appears separately from the linear part, we obtain

$$
\frac{d \vec{R}}{d \tau}=\mathrm{L} \vec{R}+\vec{N}
$$

where

$$
\vec{R}=\left(\begin{array}{c}
X \\
Y \\
Z
\end{array}\right)
$$




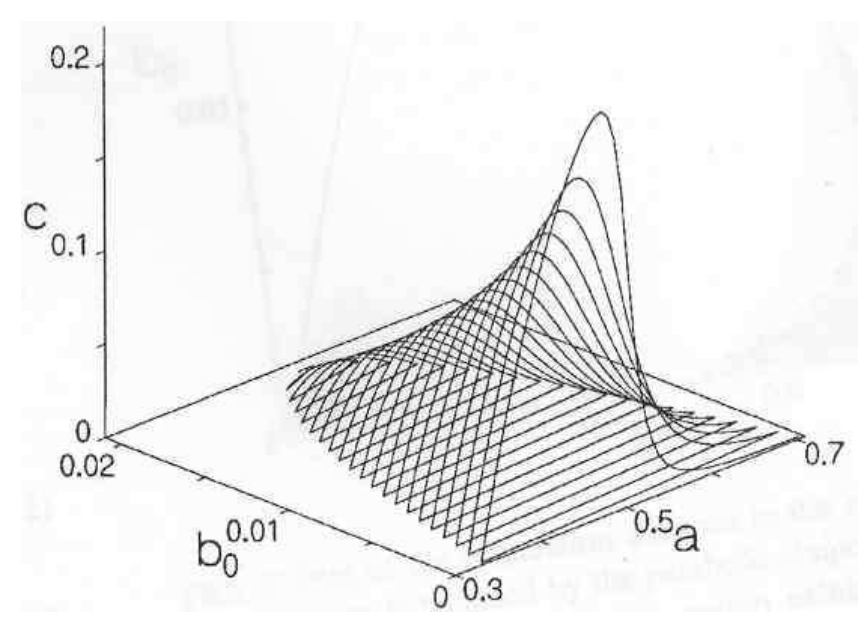

Figure 1: The instability region in the $a, b_{0}$ abd $c$ parameter space. It is bounded by three surfaces, namely, the $c_{0}$ surface shown by a series of curved lines, and $c=0$ plane and $b_{0}=0$ plane.

$$
\mathbf{L}=\left(\begin{array}{ccc}
-\alpha & -\chi & 0 \\
-b_{0} \Gamma & -b_{0} \delta & a b_{0} \\
c & 0 & -c
\end{array}\right)
$$

and the nonlinear part $\vec{N}$ is given by

$$
\vec{N}=\left(\begin{array}{c}
-b_{0} X^{2}-X Y \\
b_{0}\left(b_{0} X^{2}-X Y\right) \\
0
\end{array}\right) .
$$

As we are interested in series expansion around the point of Hopf bifurcation, we consider the stability of the fi xed point as a function of the parameter $c$. This is done by finding the value of $c=c_{0}$ at which the real part of the complex eigen value of the stability of the matrix $\mathbf{L}$ vanishes.

Since $c$ is non-negative, we obtain a unique $c_{0}$ for the allowed pair of $a$ and $b_{0}$ values within the instability. Figure 1 shows a three-dimensional plot of the instability region involving all the three parameters of the model.

To obtain an approximate analytical solution of Eq. (5.8), we follow a reductive perturbative approach similar to that used in Refs. [33] and [34]. We choose $c=c_{0}(1-\varepsilon)$ with $0<\varepsilon \ll 1$, and write the matrix $\mathbf{L}$ as a sum of two matrices, $\mathbf{L}=\mathbf{L}_{\mathbf{0}}+\varepsilon \mathbf{L}_{\mathbf{1}}$, where $\mathbf{L}_{\mathbf{0}}$ is the matrix $\mathbf{L}$ evaluated for $c=c_{0}$, and

$$
\mathbf{L}_{\mathbf{1}} \equiv\left(\begin{array}{ccc}
0 & 0 & 0 \\
0 & 0 & 0 \\
-c_{0} & 0 & c_{0}
\end{array}\right)
$$

The eigenvalues of $\mathbf{L}_{\mathbf{0}}$ are

$$
\lambda_{1,-1}= \pm i \omega \text { and } \lambda_{0}=T
$$

where $\omega^{2}=P$, where $P$ and $T$ are sum of the eigen values, and sum of the product of the pairs of eigen values evaluated at $c=c_{0}$. Taking the solution for $\vec{R}$ as a growth out of the critical 


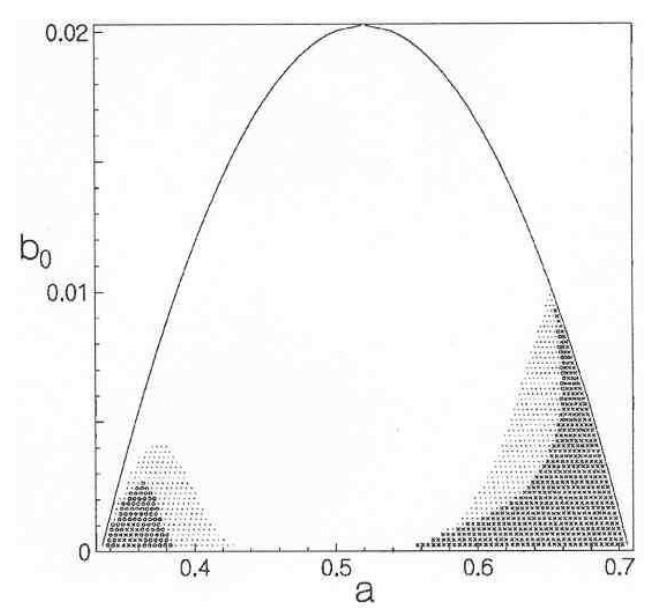

Figure 2: A plot of the bifurcation diagram of in the $a-b_{0}$ plane. The instability region is bounded by nearly parabolic curve and $b_{0}=0$ line. The unshaded (shaded) region is the supercritical (subcritcal) bifurcation. The light and dark shaded regions refer to the regions where the quintic and septic amplitude equations hold.

eigenmodes, we express it as a linear combination of these eigenmodes:

$$
\vec{R}(\tau)=\Psi e^{i \omega \tau} \vec{r}_{1}+\Psi_{0} e^{\lambda_{0} \tau} \vec{r}_{0}+\Psi^{*} e^{-i \omega \tau} \vec{r}_{1}^{*}=\sum_{j=1}^{-1} \Psi_{j} e^{\lambda_{j} \tau} \vec{r}_{j}
$$

where $\vec{r}_{j}$ 's are right eigenvectors defi ned by $\mathbf{L}_{0} \vec{r}_{j}=\lambda_{j} \vec{r}_{j}$ with $\vec{r}_{-1}=\vec{r}_{1}^{*}$. We also introduce left eigenvectors, $\vec{s}_{j}^{T}$, defi ned by $\vec{s}_{j}^{T} \mathbf{L}_{\mathbf{0}}=\lambda_{j} \vec{s}_{j}^{T}$, where $T$ stands for the transpose. Substituting this expression for $\vec{R}$ in the matrix equation, Eq.(5.8), and multiplying both sides of the equation by one of the left eigenvectors, we obtain an equation governing the corresponding amplitude:

$$
e^{\lambda_{j} \tau} \frac{d \Psi_{j}}{d \tau}=\varepsilon \sum_{k} \mu_{j k} \Psi_{k} e^{\lambda_{k} \tau}+\sum_{l, m, m \leq l} g_{j l m} \Psi_{l} \Psi_{m} e^{\left(\lambda_{l}+\lambda_{m}\right) \tau}
$$

Expressions for the coeffi cients $\mu_{j k}$ and $g_{j l m}$ are given in Ref. [24].

We express $\Psi_{j}$ as a power series expansion in $\varepsilon^{1 / 2}$ :

$$
\Psi_{j}=\varepsilon^{1 / 2} \psi_{j}^{(1)}+\varepsilon \psi_{j}^{(2)}+\varepsilon^{3 / 2} \psi_{j}^{(3)}+\cdots
$$

and introduce multiple time scales such that

$$
\frac{d}{d \tau}=\frac{\partial}{\partial \tau}+\varepsilon \frac{\partial}{\partial \tau_{1}}+\varepsilon^{2} \frac{\partial}{\partial \tau_{2}}+\cdots
$$

where $\tau_{1}=\varepsilon \tau, \tau_{2}=\varepsilon^{2} \tau, \cdots$. Substituting these expressions for $\Psi_{j}$ and $d / d \tau$ into the equation for the amplitudes, Eq.(5.15), we successively solve by equating terms of the same order in powers of $\varepsilon$. First, terms of $\mathscr{O}\left(\varepsilon^{1 / 2}\right)$ give

$$
\frac{\partial \psi_{j}^{(1)}}{\partial \tau}=0
$$


implying that $\psi_{j}^{(1)}$ is constant in the time scale of $\tau . \mathscr{O}(\varepsilon)$ terms give the equation

$$
\frac{\partial \psi_{j}^{(2)}}{\partial \tau}=\sum_{k, l, l \leq k} g_{j k l} \psi_{k}^{(1)} \psi_{l}^{(1)} e^{\left(\lambda_{k}+\lambda_{l}-\lambda_{j}\right) \tau}
$$

which, upon integration, gives

$$
\psi_{j}^{(2)} e^{\lambda_{j} \tau}=\sum_{k, l, l \leq k} h_{j k l} \psi_{k}^{(1)} \psi_{l}^{(1)} e^{\left(\lambda_{k}+\lambda_{l}\right) \tau}
$$

where $h_{j k l}=g_{j k l} /\left(\lambda_{k}+\lambda_{l}-\lambda_{j}\right) . \mathscr{O}\left(\varepsilon^{3 / 2}\right)$ terms give the equation

$$
\frac{\partial \psi_{j}^{(3)}}{\partial \tau}+\frac{\partial \psi_{j}^{(1)}}{\partial \tau_{1}}=\sum_{k} \mu_{j k} \psi_{k}^{(1)} e^{\left(\lambda_{k}-\lambda_{j}\right) \tau}+\sum_{k, l, l \leq k} g_{j k l}\left(\psi_{k}^{(1)} \psi_{l}^{(2)}+\psi_{k}^{(2)} \psi_{l}^{(1)}\right) e^{\left(\lambda_{k}+\lambda_{l}-\lambda_{j}\right) \tau}
$$

where $g_{j k l}$ have been given in Ref. [24]. Using the compatibility condition, we match terms that are varying on a slow time scale found on both sides of the equality, and extract the slow dynamics

$$
\frac{\partial \psi_{j}^{(1)}}{\partial \tau_{1}}=\mu_{j j} \psi_{j}^{(1)}+\eta_{j}\left|\psi_{1}^{(1)}\right|^{2} \psi_{j}^{(1)}
$$

An expression for $\eta_{j}$ is given in [24]. (The subscript $\mathrm{j}=1$ is left out from $\Psi_{1}$ for the sake of brevity.) To $\mathscr{O}\left(\varepsilon^{1 / 2}\right), \Psi=\varepsilon^{1 / 2} \psi^{(1)}$ and, thus, Eq.(5.15) takes the form of a cubic Stuart-Landau equation:

$$
\frac{d \Psi}{d \tau}=\varepsilon \mu \Psi+\eta|\Psi|^{2} \Psi
$$

Note that $\mu$ and $\eta$ are complex coeffi cients. $\Psi$ is the complex order parameter given by $\Psi=$ $|\Psi| e^{i \Omega \tau}$. Both the amplitude and the frequency $\Omega$ are easily determined.

This solution exists provided $\eta$ is negative since $\mu$ is positive. $\eta$ is found to be negative over a major part of the instability region in the $b_{0}-a$ plane, as shown in Fig. 2 (the unshaded region). In this case, since the amplitude of the order parameter grows continuously in proportion to $\varepsilon^{1 / 2}$, the transition is continuous (a second-order-type transition) corresponding to supercritical bifurcation. However, there is a relatively small portion of the instability region, shown in the same fi gure in different shades, where $\eta$ is found to be positive implying that the transition is discontinuous corresponding to supercritical bifurcation. In this regime, one has to go to quintic or even higher orders in the amplitude equation to obtain an expression for the order parameter. In fact order parameter equation upto septic order has been derived [24] that covers the whole parameter space (Fig. 2). The solutions obtained from the above equations agree well with the numerical solution obtained by solving Eqs. (5.1-5.3). (See for details Ref.[24].)

The above analysis shows that the bistable nature of amplitudes of various densities arises due to the forward Hopf bifurcation. In the parameter space of $a-b_{0}$ (see Fig. 2), large regions correspond to supercritical bifurcation where the amplitude grows smoothly. However, for low values of $b_{0}$, there is region of $a$ values for which the bifurcation is subcritical. For such parameter values, the bistable nature of densities are abrupt across the transition. It is this region that would be relevant to experimental situation. One more comment that may be useful to the dislocation community. The present exercise shows that a 'free energy like potential' function can be derived 
in the neighborhood of the bifurcation point. However, as the derivation is valid only in the vicinity of bifurcation point, the nature of solution obtained from this analysis cannot be pushed deep into the limit of instability. Finally, in principle, a similar analysis can be carried out for the full set of four variables with applied strain rate as the drive parameter. However, it is clear that the present analysis already shows that there would be stress drops due to bistable nature of the densities.

\section{NEGATIVE STRAIN RATE SENSITIVITY}

As mentioned in the introduction, the collective pinning and unpinning of dislocations leads to the negative strain rate behavior of the flow stress. The fact that the negative SRS branch cannot be measured in a strict sense is recognized, but the presence of this branch clearly shows up in the dynamics of the PLC effect. Even so, early formulations and the way experimental measurements have been carried out has given rise to considerable confusion. Here we discuss briefly the concept of negative SRS and working methods adopted in the literature, and also provide a dynamical interpretation of negative SRS wherein a clear connection will be established with the slow manifold of the AK model.

Penning [7] was the fi rst to recognise the necessity of negative SRS in the PLC effect. Theories of dynamic strain aging assume that the interaction of dislocations with solute atoms when averaged over the specimen dimensions can be represented by a constitutive relation connecting stress, strain, and strain rate which is conventionally written as [7]

$$
\sigma=h \varepsilon+F(\dot{\varepsilon})
$$

The basic assumption inherent in Eq. (6.1) is that stress can be split into a sum of two functions, one of which is a function of $\varepsilon$ alone and the other function of $\dot{\varepsilon}$ alone. Then, the SRS is defi ned as

$$
\mathscr{S}=\left.\frac{\partial \sigma}{\partial \ln \dot{\varepsilon}}\right|_{\varepsilon}=\dot{\varepsilon} \frac{d \sigma}{d \dot{\varepsilon}}
$$

Clearly, this defi nition uses $\varepsilon, \sigma$ and $\dot{\varepsilon}$ as a state variables even though these variables are history dependent. At a working level, however, strain is fi xed at a small nominal value and the flow stress at that value is used to obtain the SRS [39].

To begin with, we recall how the negative SRS is 'measured' in experiments on the PLC effect. There are a few attempts to 'measure' the unstable branch as a function of the strain rate [39], even though there is a full recognition of the limitations of such a measurement. Here we describe the procedure adopted by Kubin's group [40]. Following the decomposition of Pennings, the strain is fi xed at $\varepsilon=8 \times 10^{-2}$ and the mean of the upper stress values of the serrations is taken to represent the unstable branch. (See for more details in Ref. [40].) A plot of the flow stress as a function of the strain rate is shown in Fig. 3b. The alloy used is $A l-5 \% \mathrm{Mg}$ tested at $300 \mathrm{~K}$. Note the logarithmic scale along the $\mathrm{x}$-axis.

Our approach is based on the relaxation oscillations inherent to the dynamics of the PLC effect. In this picture one concludes the existence of the unstable branch on the basis of strain bursts, but one never records any points in this region. This is suitable point of view for our study as we will use a method that provides a formal basis for the relaxation oscillations arising in the model. 

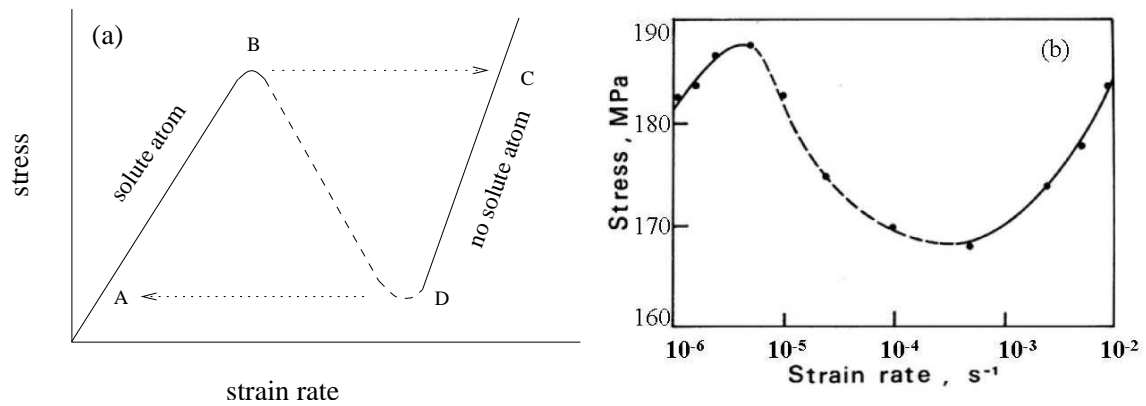

Figure 3: (a) A schematic plot of the fbw stress $v s$ strain rate in case of the PLC. (b) The fbw stress at strain $\varepsilon=8 \times 10^{-2}$ for $A l-5 \% M g$ tested at $300 \mathrm{~K}$ under constant strain rate [40].

\subsection{Slow Manifold Analysis}

Within the scope of the AK model, while the negative SRS was numerically determined quite early [13], analytical approach was provided by Rajesh and Ananthakrishna [12] in terms of the structure of the slow manifold of the model [27]. The methodology of slow manifold analysis is basically a "dimensional reduction" procedure that provides a smaller dimensional version of the original dynamical system that retains the essential dynamics. This is best suited for analysis of nonlinear slow-fast dynamical systems wherein all trajectories are attracted, in the long time limit, to a subspace of $\mathbb{R}^{n}$ which forms a topological invariant manifold.

The geometry of the slow manifold of the original model has been analyzed in detail [27, 12]. The analysis shows that the relaxational nature of the PLC effect arises from the atypical bent nature of the manifold. Here we recall some relevant results on the slow manifold of the original model $(D=0)$. (We shall later extend the ideas to the situation when the spatial degrees of freedom are switched on.) Slow manifold expresses the fast variable in terms of the slow variables. At a working force this is done by setting the derivative of the fast variable to zero [12, 27]

$$
\dot{\rho}_{m}=g\left(\rho_{m}, \phi\right)=-b_{0} \rho_{m}^{2}+\rho_{m} \delta+\rho_{i m}=0 .
$$

where $\delta=\phi^{m}-\rho_{i m}-a$. The variable $\delta$ has been shown to have all the features of an effective stress and thus plays an important physical role [27], particularly in studying the pinning-unpinning of dislocations. We note that $\delta$ is a combination of two slow variables $\phi$ and $\rho_{\text {im }}$ both of which take small positive values. Hence, $\delta$ takes on small positive and negative values. Using Eq. (6.3), we get two solutions

$$
\rho_{m}=\left[\delta+\left(\delta^{2}+4 b_{0} \rho_{i m}\right)^{1 / 2}\right] / 2 b_{0},
$$

one for $\delta<0$ and another $\delta>0$. For the region $\delta<0$, as $b_{0}$ is small $\sim 10^{-4}$, we get $\rho_{m} / \rho_{\text {im }} \approx$ $-1 / \delta$ which takes on small values. On the other hand, when $\delta>0, \rho_{m} \approx \delta / b_{0}$ which is large. These two regions corresponds to different parts of the slow manifold. A plot of the slow manifold in the $\delta-\rho_{m}$ plane is shown in Fig. 4a. For the sake of illustration, we have plotted a monoperiodic trajectory describing the changes in the mobile density during a loading-unloading cycle. The inset in Fig. 4a shows $\rho_{m}(t)$ and $\phi(t)$. As can be seen, there is region where the ratio of the mobile to immobile density is small $\rho_{m} / \rho_{i m} \approx-1 / \delta$ and negative which is marked as $S_{2}$. As the $\rho_{m}$ is small, $S_{2}$ can be identifi ed with the 'pinned state of dislocations'. In contrast, for positive values of $\delta$, as 

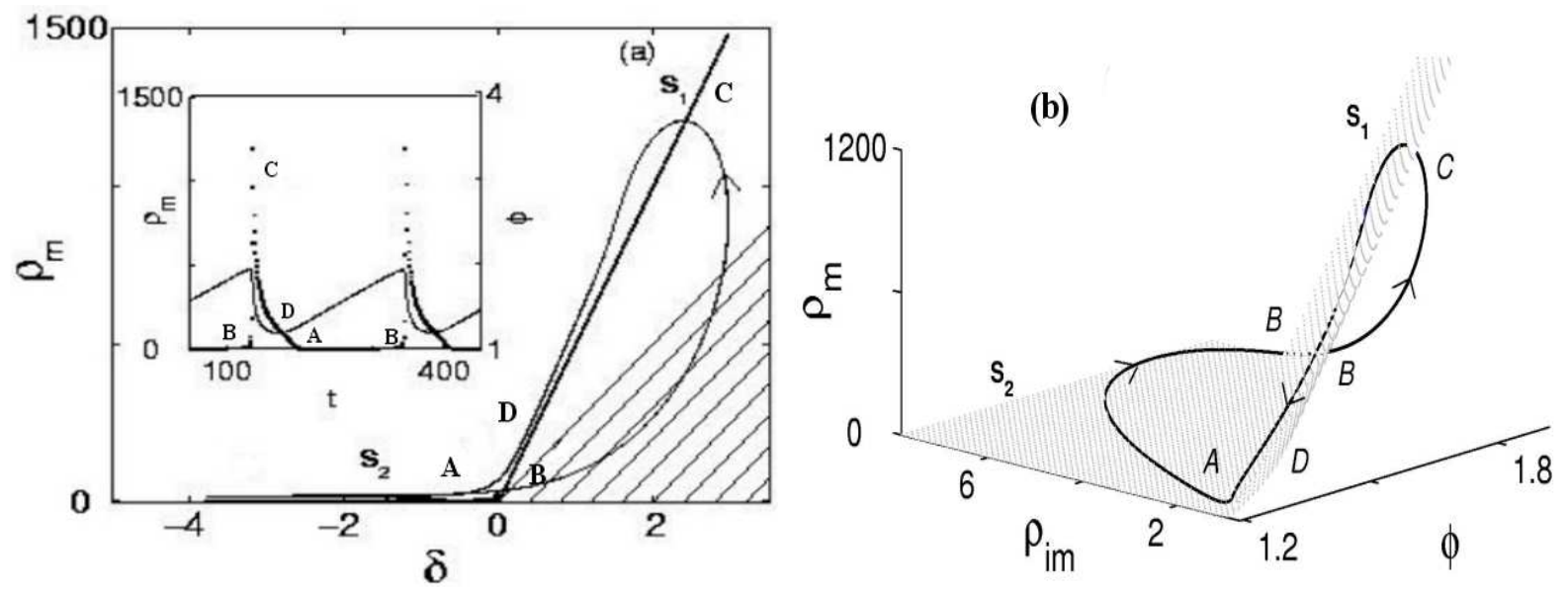

Figure 4: (a)Bent slow manifold $S_{1}$ and $S_{2}$ (thick lines) with a simple trajectory for $\dot{\varepsilon}=200$ and $m=3$. Inset: $\rho_{m}$ (dotted curve) and $\phi$ (solid line). (b)The same trajectory in the $\left(\phi, \rho_{i m}, \rho_{m}\right)$ space.

$\rho_{m}$ is large, we refer to it as the 'unpinned state of dislocations'. These two pieces $S_{2}$ and $S_{1}$ are separated by $\delta=0$, which we refer to as the fold line [12,27]. For completeness, the corresponding plot of the slow manifold in the $\left(\rho_{m}, \rho_{i m}, \phi\right)$ space is shown in Fig. $4 \mathrm{~b}$, along with the trajectory and the corresponding symbols. In this space, one can see that $\delta=\phi^{m}-\rho_{i m}-a=0$ is a line that separates the pieces $S_{2}$ and $S_{1}$ of the slow manifold, and hence the name fold line.

We shall map the various regions in Fig. 4b with that in Fig. $4 \mathrm{a}$ as the latter will be convenient for studying dislocation confi gurations later. In Fig. 4b, the trajectory enters $S_{2}$ at $A$ and leaves at $B$. For this part of trajectory, the value of $\delta$ (in Fig. 4a) decreases from zero to a maximum negative value and reverts to zero value. Beyond this point as the trajectory leaves $S_{2}$ in Fig. $4 \mathrm{~b}, \delta$ becomes positive. The corresponding points are marked on both the fi gures. In addition, in the inset of Fig. 4a, we have shown the correspondence with $\rho_{m}$. The segment $A B$ in Fig. $4 \mathrm{~b}$ can be identifi ed with the flat region of $\rho_{m}(t)$ in the inset of Fig. 4a with the same symbol. As the trajectory crosses $\delta=0, \partial g / \partial \rho_{m}$ becomes positive and it accelerates into the shaded region (Fig. 4a) rapidly till it reaches $\rho_{m}=\delta / 2 b_{0}$ ( point $C$ ). Thereafter it settles down quickly on $S_{1}$ decreasing to $D$ rapidly till it reenters $S_{2}$ again at $A$. The burst in $\rho_{m}$ (inset in Fig. 4a) corresponds to the segment $B C D A$ in Figs. $4 \mathrm{a}$ and $\mathrm{b}$. The nature of trajectories for higher strain rate remain essentially the same, but are chaotic.

\subsection{Connection to negative SRS}

The above discussion on the slow manifold of the model demonstrates how to separate out different dislocation mechanisms contribute to the time development of the variables. This should therefore help us to set up a correspondence between the stress-strain rate space and the slow manifold (Figs. 4 a,b). Having identifi ed the regions of the slow manifold with the pinned and unpinned states of dislocations, we now consider the variation of stress when dislocations are pinned and are unpinned. First consider Eq. 2.4 for $D=0$ which reduces to

$$
\dot{\phi}=d\left[\dot{\varepsilon}-\dot{\varepsilon}_{p}\right]
$$




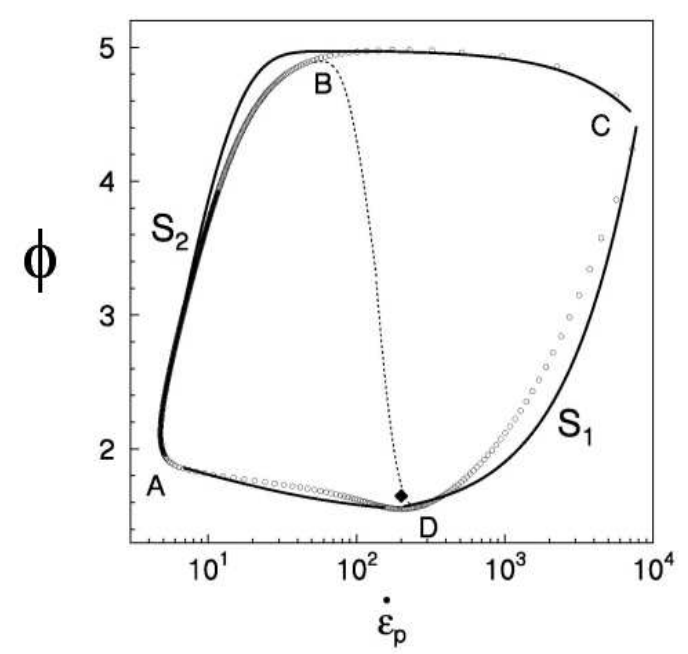

Figure 5: The empty circles show the phase space projection of stress $\sigma v s$. strain rate $\dot{\varepsilon}_{p}$. The dotted line represents the negative strain rate sensitivity (SRS) region. The thick lines are analytical approximations of corresponding regions (Ref. [27]).

where $\dot{\varepsilon}_{p}=\phi^{m} \rho_{m}$ defi nes the plastic strain rate. We shall now calculate strain rate sensitivity numerically using Eqns. (2.1 - 2.4). This is shown in Fig. 5 (o). From our earlier discussion, we know that when the trajectory is on $S_{2}, \rho_{m}$ is nearly constant and small in magnitude. As this implies a pinned confi guration, according to Eqs. $(2.1-2.4), \phi$ should increase monotonically and hence the segment $\mathrm{AB}$ on $S_{2}$ in Fig. 4 corresponds the rising branch $A B$ in Fig. 5. For this branch, one can easily see that the (mean) value of $S \sim 3.5$ using Eq. (6.2). Further, at the point where the trajectory leaves $S_{2}$ part of the slow manifold, namely $B$ in Fig. 4b, the value of $\delta$ approaches zero (Fig. 4a), and correspondingly $\phi$ reaches its maximum value. Once the trajectory leaves $S_{2}$ and jumps to $S_{1}, \rho_{m}$ increases abruptly outside $S_{2}$ (see $B C$ in the inset of Fig. 4 a)), $\delta=0$ line separates the pinned state from the unpinned state. Thus, $\delta=0$ physically corresponds to the value of the effective stress at which dislocations are unpinned. This evidently corresponds to the strain rate jump from $B$ to $C$ in Fig. 5. Note that the slope $\partial \phi / \partial \dot{\varepsilon}_{p}$ for this portion of the orbit is negative and quite small negative unlike the zero value for the equivalent part in Fig. 3. Further, we know from Fig. 4, once the trajectory reaches $S_{1}$, the value of $\rho_{m}$ decreases rapidly resulting in the decrease of $\dot{\varepsilon}_{p}$. Thus, the region CD in Fig. 5 corresponds to $C D$ segment of the trajectory on $S_{1}$ in Fig. 4 ( or inset of 4a). For this branch, one can quickly check that the strain rate sensitivity is positive, having a mean value $(\sim 1.5)$ which is a factor of 2 less than that for the branch $A B$, implying that the nature of dissipation is quite different from that operating on $A B$. This is consistent with known facts about the two branches as mentioned in the introduction. Combining this with the fact that $\dot{\rho}_{m}$ is decreasing, the branch $C D$ in Fig. 5 can be identifi ed with the slowing down of the mobile dislocations.

The above picture can be made more concrete by actually calculating stress as a function of strain rate. This can be done analytically as approximate equations of motion for each part of the slow manifold $A B$ on $S_{2}, B C$ outside the slow manifold, $C D$ on $S_{1}$, and unstable $D A$ corresponding to the jump between the two pieces of the slow manifold are known. Now consider, the equation 
for $\dot{\varepsilon}_{p}$ given by

$$
\frac{d \dot{\varepsilon}_{p}}{d t}=\rho_{m} m \phi^{m-1} \dot{\phi}+\phi^{m} \dot{\rho}_{m}
$$

which on using Eqs. (2.1) and (2.4) gives

$$
\frac{d \dot{\varepsilon}_{p}}{d \phi}=\frac{\dot{\varepsilon_{p}}\left(\frac{m \dot{\varepsilon} d}{\phi}+\delta\right)-\dot{\varepsilon}_{p}^{2}\left(\frac{m d}{\phi}+\frac{b_{0}}{\phi^{m}}\right)+\rho_{i m} \phi^{m}}{d\left(\dot{\varepsilon}-\dot{\varepsilon_{p}}\right)}
$$

Note that in the slow manifold description, all slow variables appear as parameters. However, since SRS describes the dependence of the slow variable $\phi$ as a function of the (derived) fast variable $\dot{\varepsilon}_{p}$, we will consider the other two variables $\rho_{\text {im }}$ or $\delta$ or both as parameters. Our interest here is to obtain approximate expressions for $\dot{\varepsilon}_{p}(\phi)$ on different branches. To do this, we use typical values of $\delta$ and $\rho_{i m}$ for the interval under question. As stated earlier, the trajectory has different dynamics in different regions of the slow manifold. These are (a) on $S_{2}$ where $\dot{\rho}_{m}$ is nearly zero for the entire time spent by the trajectory on $S_{2}$, (b) just outside $S_{2}$ where $\dot{\rho}_{m} \sim \rho_{m} \delta$, (c) on $S_{1}$ where $\rho_{m} \sim \delta b_{0}$ for $\dot{\varepsilon}_{p}>\dot{\varepsilon}$, and (d) when the trajectory jumps from $S_{1}$ to $S_{2}$. Approximate solutions obtained for these cases are shown in the $\phi-\dot{\varepsilon}_{p}$ plot by solid lines. (For details see [27].) It is clear that the approximate solutions shown by the bold lines are quite similar to numerically exact result shown in Fig. 5 by o.

Now it is possible study the qualitative changes that ensue as the applied strain rate is increased. In our model, for a region of parameters $a, b_{0}$, there is region for small $\dot{\varepsilon}$ where the amplitude of the stress drops increases, and thereafter it decreases. This feature is a direct result of the existence of back-to-back Hopf bifurcations in the model (see [23]). On the other hand, experimentally one sees only a decreasing trend. While the decreasing trend is consistent, the increasing trend seen in the model for low strain values can be traced to the effect of another crucial parameter in the model, namely, $b_{0}$, which corresponds to the remobilization of immobile dislocations. As demonstrated in the previous section, in our model, a large part of $a-b_{0}$ parameter space is supercritical Hopf bifurcation, in particular large values of $b_{0}$ ( see Fig. 2). This is the region in which one sees increasing stress drop for small $\dot{\varepsilon}$. However, there is a region of small values of $b_{0}$, where the bifurcation from the steady state is subcritical, i.e., across the transition, the amplitude of the stress change is abrupt. Clearly, it is possible to choose values of $b_{0}$ where this jump can be made suffi ciently large in which case the amplitude of the stress drops can be made to decrease with $\dot{\varepsilon}$ right from the onset of the PLC effect as seen in experiments.

\section{Visualization of dislocation configurations}

When the spatial degrees of freedom are included, there is no additional complication as the slow manifold is defi ned at each point. In this case, a convenient set of variables for visualization of dislocations is $\left(\rho_{m}(x), \delta(x), x\right)$. Here, we recall that we have shown that the model successfully reproduces the crossover from a low dimensional chaotic state at medium and low strain rates to a power law state of stress drops seen at high strain rates [23]. The former corresponds to type 
$\mathrm{C}$ and $\mathrm{B}$ bands and latter to the propagating type A band [19]. If the difference in the dynamics in these regimes of strain rate is the underlying cause for the different types of bands seen with increasing strain rate, it would be nice to visualize the confi guration of dislocations in the respective regimes. Thus, our aim is to investigate the nature of typical spatial confi gurations in the chaotic and the power law regimes of stress drops and study the changes as we increase the strain rate. For simplicity, we shall use $h=0$ for which we have $\phi_{e f f}=\phi$. ( It is straightforward to extend the arguments to the case when $h \neq 0$.) Then, the plastic strain rate $\dot{\varepsilon}_{p}(t)$ is given by

$$
\dot{\varepsilon}_{p}(t)=\phi^{m}(t) \frac{1}{l} \int_{0}^{l} \rho_{m}(x, t) d x=\phi^{m}(t) \bar{\rho}_{m}(t)
$$

where $\bar{\rho}_{m}(t)$ is the mean mobile density $\left(=\sum_{j} \rho_{m}(j, t) / N\right.$ in the discretized form). With the inclusion of spatial degrees of freedom, the yield drop is controlled by the spatial average $\bar{\rho}_{m}(t)$ rather than by individual values of $\rho_{m}(j)$. Further, we note that the confi guration of dislocations change during one loading-unloading cycle. However, one should expect that confi gurations will be representative for a given strain rate. Further, we know that the drastic changes occurs during an yield drop when $\bar{\rho}_{m}(t)$ grows rapidly. Thus, we focus our attention on the spatial confi gurations on the slow manifold at the onset and at the end of typical yield drops.

First consider the confi guration seen just before and after the yield drop when the strain rate is in the chaotic regime. In this regime, the stress drop magnitudes are large which implies that the change in mobile density is large. Figures $6 \mathrm{a}$, b for a typical value of $\dot{\varepsilon}=120$. It is clear that both at the onset and at the end of a typical large yield drop, the $\delta(j)$ values which reflect the state of system ( pinned or unpinned state), is negative and correspondingly the mobile density $\rho_{m}(j)$ 's are small, i.e., most dislocations are in a strongly pinned state. ( Recall that $\delta$ signifi es how close the spatial elements are close to unpinning threshold.) The arrows show the increase in $\rho_{m}(j)$ at the end of the yield drop. We have checked that this is a general feature for all yield drops in the chaotic regime of strain rates. Now consider dislocation confi guration in the scaling regime at high strain rates, say, $\dot{\varepsilon}=280$, at the onset and at the end of an yield drop shown in Fig. $6 \mathrm{c}$,d respectively. In contrast to the chaotic regime, in the scaling regime, most dislocations are clearly seen to be at the threshold of unpinning with $\delta(j) \approx 0$, both at the onset and end of the yield drop. This also implies that they remain close to this threshold all through the process of an stress drop. We have verifi ed that the edge-of-unpinning picture is valid in the entire power law regime of stress drops for a range of $N$ values. Further, as a function strain rate, we fi nd that the number of spatial elements reaching the threshold of unpinning $\delta=0$ during an yield drop increases as we approach the scaling regime.

\section{Summary and Conclusions}

We fi rst summarize and make appropriate comments wherever necessary. We have shown that established methods of analysis in the area of dynamical systems can be gainfully employed to get a good insight the PLC effect. The fi rst question we have addressed is the mathematical mechanism leading to the bistable nature of dislocation densities which translates to two levels of stress values seen at low strain rates. In dynamical approaches, the order parameter fi elds are the slow modes. The reductive perturbative approach used is a method wherein fast modes are enslaved by the slow 

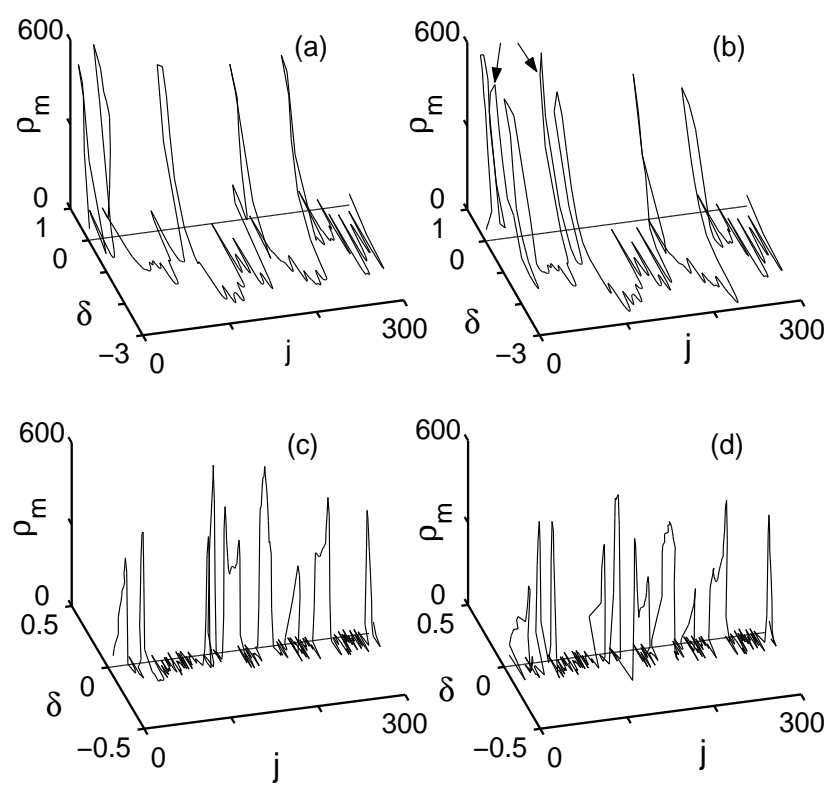

Figure 6: Dislocation configurations on the slow manifold at the inset and at the end of an yield drop: (a) and (b) for $\dot{\varepsilon}=120$ (chaotic regime), and (c) and ( d) for $\dot{\varepsilon}=280$ (scaling regime).

modes in a systematic manner. The resulting equations are for the order parameter variables which in the present context refers to the amplitude and phase of the limit cycle solutions. This also means that one associates a free energy like function in the neighborhood of the bifurcation point.

The next question that we have addressed is the origin of negative strain rate sensitivity of the flow stress, which in general terms also means bistable strain rate values. The idea is that different parts of phase space can be essentially described by approximate dynamics wherein a few dislocation mechanisms play a dominant role which is well captured by an analysis of the slow manifold. In the case of the AK model, the slow manifold have two distinct branches, one where the dislocations are in the pinned state and another where they are in the unpinned state. Approximate equations of motions are fi rst obtained from the original complicated coupled equations. Using this, each branch of the SRS is then calculated. The interpretation is that the unstable branch corresponds to the unstable manifold of the system of equations on which the unstable fi xed point is situated.

One other advantage of the slow manifold description is that it is particularly useful in giving a geometrical picture of the spatial confi gurations. The crucial parameter for the description is $\delta$ which has all features of effective stress of unpinning dislocation. This method also helps us to visualize dislocation confi gurations in different regimes of strain rates, for example, type $\mathrm{C}, \mathrm{B}$ and A. The fi rst two have been earlier identifi ed with the chaotic state where as the type A with the power law regime of stress drops $[19,23]$. The study shows that the confi gurations of dislocations that are largely in the pinned state in low and medium strain rates (chaotic domain) are pushed to the threshold of unpinning as we increase strain rate (power law stress drop regime).

Finally, this methodology offers a dynamical reason for the smallness of the yield drops in the high strain rate region of band A [12, 27]. Indeed, we have shown that this is a direct result of the existence of a reverse Hopf bifurcation at high strain rates [23, 12, 27]. In this regime, due 
to softening of the eigen values (as a function of the applied strain rate), the orbits are mostly restricted to the region around the saddle node fi xed point located on the $S_{1}$ part of the manifold. Note also that there is a dynamic feed back between the stress determined by Eq. 2.4 and the production of dislocations in Eq. 2.1 which provides an explanation for the slowing down of the plastic relaxation. This sets up a competition between the time scale of internal relaxation and the time scale determined by the applied strain rate (essentially Deborah number). We note that while the time scale for internal relaxation is increasing, that due to the applied strain rate is decreasing.

Regarding the spatial features seen in the model, we stress that all these features emerge purely due to dynamical reasons without any recourse to using the negative strain rate sensitivity feature as an input, as is the case in most models [41, 42, 43, 44]. Even the recently introduced polycrystalline plasticity model which reproduces the crossover behavior also uses the negative SRS as an input [45]. The dynamical approach followed here clearly exposes how the slowing down of the plastic relaxation occurs due to a feed back mechanism of dislocation multiplication and applied strain rate as we reach the power law regime of stress drops.

Acknowledgment: The author wishes to acknowledge the award of Raja Ramanna Fellowship.

\section{References}

[1] L.P. Kubin, C. Fressengeas and G. Ananthakrishna, in Collective Behaviour of Dislocations, edited by F.R.N. Nabarro and M.S. Deusbery, Dislocations in Solids Vol.11. P 101, North-Holland, Amsterdam, 2002.

[2] F. Le Chatelier, Influence du temps et de la temperature sur les essais au choc, Rev. de Métall., 6, 914 (1909); A. Portevin and Le Chatelier, Sur un phénomène observe lors de l'essai de traction d'alliages en lours de transformation, Comptes Rendus de l'Académie des Sciences, Paris, 176, 507 (1923).

[3] M. Zaiser and P. Hähner, Oscillatory Modes of Plastic Deformation : Theoretical Concepts, Phys. Stat. Solidi B, 199, 267 (1997).

[4] A. H. Cottrell, Dislocations and Plastic Flow in Crystals, Clarendon Press, Oxford, 1953.

[5] A. Van den Beukel, Theory of the effect of dynamic strain ageing on mechanical properties, Phys. Stat. Solidi A, 30, 197 (1975).

[6] L.P. Kubin, and Y. Estrin, Portevin-Le Chatelier effect in deformation with constant stress rate, Acta. Metall., 33, 397 (1985)

[7] P. Penning, Mathematics of the Portevin-Le Chatelier effect, Acta Metall., 20, 1169 (1972)

[8] B.N.J. Persson and E. Tosatti, Physics of Sliding Friction, Kluwer Academic Publishers, Dordrecht, 1996.

[9] J.M. Carlson and J.S. Langer, Properties of Earthquakes Generated by Fault Dynamics, Phys. Rev. Lett., 62, 2632 (1989); Mechanical model of an earthquake fault, Phys. Rev. A., 40, 6470 (1989).

[10] D. Maugis and M. Barquins, Adhesion, edited by K.W. Allen, Vol. 12. Elsevier, London, 1988.

[11] G. Ananthakrishna and M.C. Valsakumar, Repeated yield drop phenomena: A temporal dissipative structure, J. Phys. D, 15, L171 (1982).

[12] S. Rajesh and G. Ananthakrishna, Relaxation oscillations and negative strain rate sensitivity in the Portevin-Le Chatelier effect, Phys. Rev. E., 61, 3664 (2000). 
Bistability, negative strain rate sensitivity and visualization of dislocation configurations.

G. Ananthakrishna

[13] G. Ananthakrishna and M.C. Valsakumar, Chaotic flow in model for repeating yielding, Phys. Lett., A95, 69 (1983).

[14] G. Ananthakrishna et al., On the existence of chaos in Jerky flow of Cu - Al alloy, Scripta. Metall., 32, 1731 (1995).

[15] S.J. Noronha, et al., Chaos in Portevin-Le Chatelier effect, Int. Jl. of Bifurcation and Chaos, 7, 2577 (1997).

[16] A. J. Lichtenberg and M. A. Libermann, Regular and Chaotic Dyanmics, Springer-Verlag, New York, 1991.

[17] H.D.I. Abarbanel, Analysis of Observed Chaotic Data, Springer-Verlag, New York, 1996.

[18] G. Ananthakrishna et al., Crossover from chaotic to self-organized critical dynamics in jerky flow of single crystals, Phys. Rev. E, 605455 (1999).

[19] M.S. Bharathi, et al., Multifractal burst in the spatio-temporal dynamics of jerky flow, Phys. Rev. Lett. 87, 165508 (2001).

[20] P. Bak, C. Tang and K. Wiesenfeld, Self-Organised Criticality : An explanation of 1/f Noise, Phys. Rev. Lett., 59, 381 (1987); Self-organised criticality, Phys. Rev. A, 38, 364 (1988).

[21] H.J. Jensen, Self-Organized Criticality, Cambridge University Press, Cambridge, 1998.

[22] G. D'Anna and F. Nori, Critical dynamics of Burst Instabilities in the Portevin-Le Chatelier Effect, Phys. Rev. Lett., 85, 4096 (2000); S. V. Franklin, F. Martens, and M. Marder, Portevin-Le Chatelier effect, Phys. Rev. E, 62, 8195 (2000); Dynamics of Plastic Deformation Fronts in an Aluminum Alloy, Phys. Rev. Lett. 78, 4502 (1997).

[23] G. Ananthakrishna and M.S. Bharathi, A dynamical approach to the spatiotemporal aspects of the Portevin-Le Chatelier effect: Chaos, turbulence and band propagation, Phys. Rev. E, 70, 026111 (2004).

[24] M. Bekele and G. Ananthakrishna, High order amplitude equation for steps on creep curve, Phys. Rev. E, 56, 6917 (1997).

[25] M.V. Glazov et al, Temporal dissipative structures in cyclically deformed metallic alloys, Appl. Phys. A, 64, 373 (1997).

[26] M. Zaiser et al, On the relations between strain and strain-rate softening phenomenon in some metalic materials : a computational study, Comp. Mater. Science, 5, 35 (1999).

[27] S. Rajesh and G. Ananthakrishna, Incomplete approach to homoclinity in a model with bent-slow manifold geometry, Physica D, 140, 193 (2000).

[28] M.S. Bharathi and G. Ananthakrishna, Chaotic and power law states in the Portevin-Le Chatelier effect, Europhys. Lett. 60, 234 (2002).

[29] A. Milik et al, Geometry of Mixed-Mode Oscillations in the 3-d Autocatalator, Int. J. of Bifurcation and Chaos, 8, 505 (1998).

[30] T. Taniuti and C.C. Wei, Reductive perturbative method in nonlinear wave propagation, J. Phys. Soc. Jpn., 24, 941 (1968).

[31] A.C. Newell and J.A. Whitehead, Finite band width, finite amplitude convection, J. Fluid Mech., 38, 279 (1968). 
[32] Y. Kuramoto and T. Tsuzuki, Reductive perturbative approach to chemical instabilities, Prog. Theor. Phys., 52, 1399 (1974).

[33] H. Mashiyama, A. Ito, and T. Ohta, Fluctuations and phase transitions far from equilibrium, Prog. Theor. Phys., 54, 1050 (1975).

[34] P.H. Richter, I. Procaccia, and J. Ross, in Advances in Chemical Physics, edited by I. Prigogine and S. Rice, Wiley Interscience, New York, 1982.

[35] L.Y. Chen, N. Goldenfeld, and Y. Oono, The renormalization group and singular perturbations : Multiple-scales, boundary layers and reductive perturbative theory, Phys.Rev. E, 54, 376 (1996).

[36] J. Carr, Applications of Center Manifold Theory, Springer-Verlag, Berlin, 1981.

[37] N. Goldenfeld, O. Martin, and Y. Oono, Intermediate asymptotics and renormalization group theory, J. Sci. Comput., 4, 355 (1989).

[38] T. Kunihiro, The renormalization group method applied to asymptoic analysis of vector fields, Prog. Theor. Phys., 94, 503 (1995) ; 97, 179 (1997).

[39] S.R. Bodner and A. Rosen, Discontinuous yielding of commercially-pure aluminium, J. Mech. Phys. Solids, 15, 63 (1967).

[40] L.P. Kubin, and Y. Estrin, A nonlinear aspect of crystal plasticity : the Portevin-Le Chatelier effect, J. de Physique, 46, 497 (1986).

[41] P. Hähner, A. Ziegenbein, E. Rizzi and H. Neuhäuser, Spatiotemporal analysis of Potevin-Le Chatelier deformation bands: Theory, simulation, and experiment, Phys. Rev. B, 65, 134109 (2002).

[42] P. G. Mc Cormick, and C. P. Ling. Numerical modeling of the Potevin-Le Chatelier effect, Acta Metall. Mater. 43,1969 (1995).

[43] S. Zhang, P. G. McCormick, Y. Estrin, The morphology of Potevin-Le Chatelier bands : Finite element simulation for Al-Mg-Si, Acta Mater., 49, 1087 (2001).

[44] M. Lebyodkin, L. Dunin-Barkowskii, Y. Bréchet, Y. Estrin, L. P. Kubin., Spatio-temporal dynamics os the Potevin-Le Chatelier effect: experiment and modelling, Acta Mater., 48, 2529 (2000) and the references therein.

[45] S. Kok, M. S. Bharathi, A. J. Beaudoin, C. Fressengeas, G. Ananthakrishna, L. P. Kubin, and M. Lybyodkin, Spatial coupling in jerky flow using polycrystal plasticity, Acta Materialia, 51, 3651 (2003). 